\title{
Ecuaciones de transporte para un gas diluido
}

\author{
Cristian Joel Ordóñez Martínez \\ Universidad Nacional Autónoma de Honduras \\ Facultad de Ciencias \\ Escuela de Física \\ cristianjoel.cjordonez@gmail.com
}

\section{Resumen}

\begin{abstract}
Se pretende detallar la teoría fundamental de la física estadística clásica, desde la idea del por que es necesario estas herramientas matemáticas para entender acerca de la dinámica de gases diluidos, se demuestra de manera alternativa el teorema de Liouville, lo cual nos es importante para poder definir la distribución de probabilidad (ensamble), para después encontrar la ecuación de Boltzmann, tomando en cuenta los encuentros moleculares, la cual nos da las soluciones acerca de los parámetros, al saber, transporte de momento, transporte de masa y transporte de energía en conjunto con el teorema de Gibbs.
\end{abstract}

\section{Conceptos BÁsicos de MECÁnica Estadística}

$\tau$ A mecánica estadística es una parte de las ciencias físicas que se encarga de determinar el comportamiento de un sistema que está constituido por muchas partículas. Trata de predecir el estado dinámico y termodinámico de un sistema macroscópico tomando solo en consideración el estado dinámico promedio de las partículas microscópicas. De esta manera la mecánica estadística se apoya en procesos estocásticos y mecánicos para su propósito [4] [5].

La justificación de utilizar esta disciplina es la dificultad en darle solución a un sistema de partículas, tomemos en cuenta la hipótesis molecular 11 solo en un mol de una sustancia existen $\sim 10^{23}$ de estas partículas. Ahora imaginémonos que queremos encontrar el estado dinámico del sistema constituido por esas partículas, según la mecánica de Newton, el movimiento de cada partícula está determinado por

$$
\frac{d \mathbf{p}}{d t}=\mathbf{F}
$$

por lo tanto necesitaríamos $\sim 10^{23}$ ecuaciones diferenciales para determinar el estado en el que se encuentra el sistema, las cuales están

\footnotetext{
${ }^{1}$ La idea en que la materia esta constituida por partículas muy pequeñas llamadas moléculas o átomos
}

acopladas entre sí, y aun mas difícil seria el introducir las condiciones iniciales (sería imposible) para encontrar la solución [5].

La mecánica estadística se construye en base al espacio fase o fásico, este es un espacio $2 N$-dimensional, con $N=l s$, donde $l$ es el número de partículas constituyentes del sistema, las cuales tienen $s$ grados de libertad, en el cual un punto en este espacio representa el estado mecánico del sistema en un instante de tiempo dado $t[5]$.

Veamos cómo es esto.

\section{Teorema de Liouville}

Supongamos un sistema constituido por $l$ particulas en el espacio tridimensional, las cuales tienen $s$ grados de libertad, denotemos con un subíndice $i$ a la $i$-ésima particula. Supongamos que cada una de estas partículas está representada por su vector de posición, y denotemos por $\mathbf{r}_{i}$ este vector de posición, de la misma manera denotemos por el vector $\mathbf{v}_{i}$ la velocidad de la partícula, según un sistema de referencia. Entonces el estado mecánico de la partícula $i$ es $\left(\mathbf{r}_{i}, \mathbf{v}_{i}\right)$ o $\left(\mathbf{r}_{i}, \mathbf{p}_{i}\right)$, donde $\mathbf{p}_{i}$ es la cantidad de movimiento lineal de la partícula igual a $m \mathbf{v}_{i}$, donde $m$ es la masa de la partícula ${ }^{2}$ [3] [1] 6].

\footnotetext{
${ }^{2}$ Suponemos que todas las partículas tienen igual masa
} 
En general, cada partícula está sometida a fuerzas externas que dan como resultado el vector $\mathbf{F}_{i}$ de fuerza en la posición de la partícula $i$ en un tiempo $t$, además las partículas interactúan unas con otras, estas interacciones que consideramos dual es de la forma $\mathbf{F}_{i j}$ (la fuerza entre la partícula $i$ debido a la partícula $j$ ) [5].

Vamos a suponer que las fuerzas que actúan sobre la partícula $i$ solo dependen de la posición en la cual se encuentra la partícula. Según la mecánica generalizada el movimiento del sistema que está constituido de $l$ partículas esta determinada por las ecuaciones de Hamilton, de la siguiente forma [4:

$$
\begin{array}{r}
\dot{q}_{j}=\frac{\partial H}{\partial p_{j}} \\
\dot{p}_{j}=-\frac{\partial H}{\partial q_{j}}
\end{array}
$$

Con $j=1,2,3, \ldots, N$, donde $q_{j}$ representa las posiciones generalizadas, $p_{j}$ representa los momentos conjugados y $H=H\left(q_{j}, p_{j}\right){ }^{3}$ es la Hamiltoneana.

En este caso denotaremos un punto de la forma $\left(q_{j}, p_{j}\right)$ el cual representa el estado mecánico en un espacio $2 N$-dimensional conocido como espacio $\Gamma$, hay que notar que

$$
\left(q_{j}, p_{j}\right)=\left(q_{1}, q_{2}, \ldots, q_{N}, p_{1}, p_{2}, \ldots, p_{N}\right)
$$

Ahora consideremos un elemento de volumen en el espacio $\Gamma$ en un tiempo especifico $t$

$$
\delta V=\delta q_{1} \delta q_{2} \ldots \delta q_{N} \delta p_{1} \delta p_{2} \ldots \delta p_{N}
$$

y definamos la densidad de puntos en este espacio como

$$
D=\frac{\delta N}{\delta V}
$$

Donde $\delta N=D \delta V$, es el número de puntos dentro del volumen elemental $\delta V$. Nos interesa encontrar la variación con respecto al tiempo del número de puntos dentro del volumen elemental. Si consideramos un estado específico $q_{j}$ y $p_{j}$, el número de puntos variará si existen flujos (denotados por $I$ ) de puntos saliendo y

\footnotetext{
${ }^{3}$ En general la Hamiltoneana depende tambien del tiempo
}

entrando de una superficie que tiene como dirección la axisa $q_{j}$ o $p_{j}$ a través de un elemento de superficie $\delta a_{q}$ o $\delta a_{p}$, respectivamente, dados como

$$
\begin{aligned}
\delta a_{q}= & \delta q_{1} \delta q_{2} \ldots \delta q_{j-1} \delta q_{j+1} \ldots \\
& \delta q_{N} \delta p_{1} \delta p_{2} \ldots \delta p_{N} \\
\delta a_{p}= & \delta q_{1} \delta q_{2} \ldots \delta q_{N} \delta p_{1} \delta p_{2} \ldots \\
& \delta p_{j-1} \delta p_{j+1} \ldots \delta p_{N}
\end{aligned}
$$

Por lo tanto la variación del número de puntos será igual a la cantidad de puntos que entran al elemento de volumen menos la cantidad de puntos que salen del elemento de volumen, a través de las caras $q_{j}$ y $q_{j}+\delta q_{j}$ como las $p_{j} \mathrm{y}$ $p_{j}+\delta p_{j}$, esto es

$$
\frac{\partial(\delta N)}{\partial t}=I_{\text {in }}-I_{\text {out }}
$$

donde

$$
\begin{aligned}
I_{\text {in }} & =I_{\text {in }-q}+I_{\text {in }-p} \\
I_{\text {out }} & =I_{\text {out }-q}+I_{\text {out }-p}
\end{aligned}
$$

sustituyendo las ecuaciones 10 y 11 en 9 , tenemos

$$
\begin{aligned}
\frac{\partial(\delta N)}{\partial t} & =\left(I_{\text {in-q }}+I_{\text {in }-p}\right)-\left(I_{\text {out }-q}+I_{\text {out }-p}\right) \\
& =\left(I_{\text {in-q }}-I_{\text {out }-q}\right)+\left(I_{\text {in }-p}-I_{\text {out }-p}\right) \\
\frac{\partial(\delta N)}{\partial t} & =\delta I_{q}+\delta I_{p}
\end{aligned}
$$

calculemos uno de ellos; es fácil encontrar que

$$
\begin{aligned}
I_{\text {out }-q}= & D\left(q_{1}, q_{2}, \ldots, q_{j}+\delta q_{j}, \ldots, q_{N}, p_{1},\right. \\
& \left.p_{2}, \ldots, p_{N}\right) \dot{q}_{j}\left(q_{1}, q_{2}, \ldots, q_{j}+\delta q_{j},\right. \\
& \left.\ldots, q_{N}, p_{1}, p_{2}, \ldots, p_{N}\right) \delta a_{q} \\
= & \delta\left(D \dot{q}_{j}\right) \delta a_{q} \\
I_{\text {in-q }}= & D\left(q_{1}, q_{2}, \ldots, q_{j}, \ldots, q_{N}, p_{1}, p_{2}, \ldots,\right. \\
& \left.p_{N}\right) \dot{q}_{j}\left(q_{1}, q_{2}, \ldots, q_{j}, \ldots, q_{N}, p_{1}, p_{2},\right. \\
= & \left.\ldots, p_{N}\right) \delta a_{q} D \dot{q}_{j} \delta a_{q}
\end{aligned}
$$

Donde $D$ y $\dot{q}_{j}$ están evaluadas en las caras correspondientes, entonces

$$
\begin{aligned}
\delta I_{q} & =I_{i n-q}-I_{\text {out }-q} \\
& =-\left[\delta\left(D \dot{q}_{j}\right)-D \dot{q}_{j}\right] \delta a_{q}
\end{aligned}
$$


si dividimos y multiplicamos por $\delta q_{j}$ y hacemos que $\delta q_{j} \rightarrow 0$ tenemos que

$$
\begin{aligned}
\delta I_{q} & =-\lim _{\delta q_{j} \rightarrow 0} \frac{\left[\delta\left(D \dot{q}_{j}\right)-D \dot{q}_{j}\right] \delta q_{j} \delta a_{q}}{\delta q_{j}} \\
& =-\lim _{\delta q_{j} \rightarrow 0}\left[\frac{\delta\left(D \dot{q}_{j}\right)-D \dot{q}_{j}}{\delta q_{j}}\right] \delta V \\
& =-\frac{\partial\left(D \dot{q}_{j}\right)}{\partial q_{j}} \delta V \\
\delta I_{q} & =-\left(D \frac{\partial \dot{q}_{j}}{\partial q_{j}}+\dot{q}_{j} \frac{\partial D}{\partial q_{j}}\right) \delta V
\end{aligned}
$$

análogamente podemos encontrar

$$
\begin{aligned}
\delta I_{p} & =I_{\text {in }-p}-I_{\text {out }-p} \\
\delta I_{p} & =-\left(D \frac{\partial \dot{p}_{j}}{\partial p_{j}}+\dot{p}_{j} \frac{\partial D}{\partial p_{j}}\right) \delta V
\end{aligned}
$$

ahora sustituyendo las ecuaciones 16 y $17 \mathrm{en}$ la ecuacion 12 tenemos

$$
\begin{aligned}
\frac{\partial(\delta N)}{\partial t}= & I_{\text {in }}-I_{\text {out }}=\delta I_{q}+\delta I_{p} \\
= & -\left(D \frac{\partial \dot{q}_{j}}{\partial q_{j}}+\dot{q}_{j} \frac{\partial D}{\partial q_{j}}\right) \delta V \\
& -\left(D \frac{\partial \dot{p}_{j}}{\partial p_{j}}+\dot{p}_{j} \frac{\partial D}{\partial p_{j}}\right) \delta V
\end{aligned}
$$

por las ecuaciones 2,3 y factorizando $\delta V$, tenemos que

$$
\begin{aligned}
\frac{\partial(\delta N)}{\partial t}= & -\left[D \frac{\partial}{\partial q_{j}}\left(\frac{\partial H}{\partial p_{j}}\right)+\frac{\partial H}{\partial p_{j}} \frac{\partial D}{\partial q_{j}}\right] \delta V \\
& -\left[D \frac{\partial}{\partial p_{j}}\left(\frac{\partial H}{\partial q_{j}}\right)-\frac{\partial H}{\partial q_{j}} \frac{\partial D}{\partial p_{j}}\right] \delta V \\
= & -\left[\frac{\partial D}{\partial q_{j}} \frac{\partial H}{\partial p_{j}}-\frac{\partial D}{\partial p_{j}} \frac{\partial H}{\partial q_{j}}\right] \delta V \\
= & -[D, H] \delta V
\end{aligned}
$$

dado que $\delta V$ es un invariante de Poincaré bajo una transformación canónica podemos escribir la ecuación anterior como

$$
\frac{1}{\delta V} \frac{\partial(\delta N)}{\partial t}=\frac{\partial(\delta N / \delta V)}{\partial t}=\frac{\partial D}{\partial t}=-[D, H]
$$

por lo tanto

$$
\frac{d D}{d t}=\frac{\partial D}{\partial t}+[D, H]=0
$$

esto indica que la variación de la densidad de puntos en el espacio $\Gamma$ no varía en el tiempo, esta propiedad de incompresibilidad de un conjunto de puntos en el espacio $\Gamma$, fue enunciado por primera vez por Liouville y se conoce como el Teorema de Liouville[2] 44.

Desde ahora denotaremos por $\delta q_{j} \delta p_{j}$ a un elemento de volumen $\delta V$ en el espacio $\Gamma$, estos tipos de volúmenes abstractos los estaremos utilizando constantemente de aquí en adelante[4].

\section{Ensamble y ecuación de Boltzmann} sin encuentros moleculares

Es importante en este punto definir un concepto referente al estudio de la mecánica estadística, el cual se conoce como ensamble y definir otra herramienta matemática que se conoce como espacio $\mu[8]$ [4].

Definamos primero el espacio $\mu$. Supongamos que estamos estudiando $N$ particulas, donde el estado dinámico de cada partícula esta determinado por $\left(\mathbf{q}_{i}, \mathbf{p}_{i}\right){ }^{4}$ donde el subíndice $i$ representa la $i$-ésima particula, es fácil darse cuenta que este objeto esta en un espacio 6 -dimensional (tres posiciones y tres momentos), a este espacio se le conoce como espacio $\mu$. Podemos notar, haciendo una comparación con el espacio $\Gamma$, que $N$ puntos en el espacio $\mu$ equivale a un punto en el espacio $\Gamma$. Mas adelante utilizaremos el espacio $\mu$ para determinar la distribución de probabilidad de una partícula, que simplemente nos habla de todos los estados dinámicos de dicha partícula [5] 8 . Ahora definamos lo que conocemos como ensamble, este concepto queda mejor determinado diciendo lo que hace, esto es: un ensamble es la medida de un conjunto de muchas replicas de un mismo sistema que son idénticos en todos los aspectos aparte de estar en diferentes estados en un instante de tiempo [8].

Supongamos que queremos saber acerca de cuantas replicas existen en un sistema, podemos pensar que solamente podríamos contar las partículas que tienen el mismo estado dinámico, pero, como lo discutimos en la introducción de este capítulo es imposible hacerlo, no podemos seguir el estado dinámico de todas las partículas, entonces debido a esta limitación solo podemos definir lo que conocemos como

\footnotetext{
${ }^{4}$ Aquí $\left(\mathbf{q}_{i}, \mathbf{p}_{i}\right)=\left(x, y, z, p_{x}, p_{y}, p_{z}\right)$, por ejemplo
} 
función de distribución de probabilidad, al saber, $f\left(q_{j}, p_{j}, t\right)$.

Que se define como

$$
f\left(q_{j}, p_{j}, t\right)=\lim _{\delta V \rightarrow 0} \frac{\delta N}{\delta V}
$$

Si observamos la definición de $f$ podemos notar que representa la probabilidad por unidad de volumen de encontrar una partícula en cierto estado dinámico, también hay que tener en cuenta que cuando hablamos de un volumen en el espacio $\Gamma$ no es un volumen real, es un volumen de puntos en dicho espacio.

Ahora podemos de esta manera contar las replicas que suceden en un conjunto de puntos, simplemente multiplicando $f$ por un estado dinámico especifico $d q_{j} d p_{j}$ en todo el espacio de configuración [2] 4].

Por la definición anterior podemos ver que $f$ es una invariante [2] [4] y que la variación en el tiempo es igual a cero, esto es

$$
\frac{d f}{d t}=\frac{\partial f}{\partial t}+\sum_{j}^{N} \frac{\partial f}{\partial q_{j}} \dot{q_{j}}+\frac{\partial f}{\partial p_{j}} \dot{p_{j}}=0
$$

Esta ecuación se le conoce como la Ecuación de Boltzmann sin colisiones, aquí asumimos que no existe interacción entre las partículas que constituyen el sistema.

\section{La jerarquía de las ecuaciones BBGKY}

Para poder conocer el estado mecánico de todo el sistema necesitamos resolver las $N$ ecuaciones acopladas anteriores, lo cual, como lo hemos estado comentando es imposible, lo que ahora necesitamos es simplificar las cosas, supongamos que queremos encontrar el posible estado mecánico de solo una partícula por unidad de volumen, supongamos la partícula 1 , esto es [8] [5] 3$]$

$$
f_{1}=\int f d q_{2} d q_{3}, \ldots, d q_{N} d p_{2} d p_{3}, \ldots, d p_{N}
$$

Esto representa la distribución de probabilidad por unidad de volumen de encontrar la partícula en cualquier estado mecánico menos en el estado mecánico 1, que es equivalente a encontrar la distribución de probabilidad por unidad de volumen de encontrar las $N-1$ partículas en el estado mecánico 1, podemos notar que existen $N$ de estas ecuaciones, pues se asume que todas las partículas son idénticas, por lo tanto la densidad de probabilidad de tener una distribución de $N$ partículas en un estado mecánico especifico por unidad de volumen es 5

$f_{1 N}=N \int f d q_{2} d q_{3}, \ldots, d q_{N} d p_{2} d p_{3}, \ldots, d p_{N}$

De la misma forma, podemos encontrar la distribución de probabilidad de tener una distribución de $N$ partículas en dos estados mecánicos diferentes, esto es

$$
\begin{aligned}
f_{12 N}= & N(N-1) \int f d q_{3} d q_{4}, \ldots, \\
& d q_{N} d p_{3} d p_{4}, \ldots, d p_{N}
\end{aligned}
$$

Podemos generar de la misma forma ecuaciones para $i$ partículas distribuidas $i-1$ en estados mecánicos diferentes, hasta encontrar la densidad de probabilidad de tener una distribución de $N$ partículas en $N-1$ estados mecánicos diferentes.

$$
f_{123 \ldots N}=N ! \int f d q_{N} d p_{N}
$$

A esta jerarquía de ecuaciones se le conocen como las ecuaciones BBGKY 6

Notemos que $f_{1 N}$ posee la información de una partícula y la función $f$ contiene la información de las $N$ partículas. También

$$
\begin{aligned}
& \frac{f_{1 N}}{N} d q_{1} d p_{1}= \\
& d q_{1} d p_{1} \int f d q_{2} d q_{3}, \ldots, d q_{N} d p_{2} d p_{3}, \ldots, d p_{N}
\end{aligned}
$$

Es la probabilidad de encontrar una partícula en el estado $d q_{1} d p_{1}$, observemos que esta probabilidad esta en el espacio $\mu$, y es mejor

\footnotetext{
${ }^{5}$ esto es debido al principio de probabilidad: si dos eventos son independientes de ocurrir al mismo tiempo, entonces la probabilidad de que ocurran simultaneamente es la suma de las probabilidades que ocurran individualmente

${ }^{6}$ Denominada así en honor de Bogoliubov, Born, Green Kirkwood e Yvon
} 
representada por $d \mathbf{q}_{1} d \mathbf{p}_{1}$.

Si las partículas son idénticas entonces la probabilidad de encontrar a las partículas en el estado $d \mathbf{q}_{1} d \mathbf{p}_{1}$ es el producto de la probabilidad de encontrar a cada partícula en el estado mecánico $d \mathbf{q}_{1} d \mathbf{p}_{1}$, esto es

$$
N\left(\frac{f_{1 N}}{N} d \mathbf{q}_{1} d \mathbf{p}_{1}\right)=f_{1 N} d \mathbf{q}_{1} d \mathbf{p}_{1}
$$

Ahora integrando en las velocidades $d \mathbf{q}_{1} f_{1 N} d \mathbf{p}_{1}$, podemos notar que

$$
\rho=\int f_{1 N} d \mathbf{p}_{1}
$$

Es la cantidad de masa por unidad de volumen "real", esto se le conoce comúnmente como densidad y

$$
n=\int f_{1 N} d \mathbf{v}_{1}
$$

Se conoce como la densidad numérica, que es el número probable de partículas por unidad de volumen, con $\rho=m n$, donde $m$ es la masa de la partícula.

\section{Ecuación de Boltzmann con en- cuentros moleculares}

Ahora daremos paso a considerar en la ecuación de Boltzmann el hecho de que existen encuentros de las partículas dentro del gas, esto lo haremos considerando la función $f$ en el espacio $\mu$. Entonces es obvio que [5] [8] [3] [1] [6] [7]

$$
\frac{d f}{d t} \neq 0
$$

Tomemos en consideración el objeto siguiente

$$
\frac{d f}{d t} d \mathbf{q} d \mathbf{p}=\frac{d f}{d t} d x d y d z d p_{x} d p_{y} d p_{z}
$$

Observemos que $d \mathbf{q} d \mathbf{p}$ representa cualquier estado dinámico de cualquier partícula, por lo tanto el objeto anterior representa la variación en el tiempo en el cual la partícula cambia su estado dinámico de uno a otro, ahora pensemos como puede variar el estado dinámico.

Supongamos que una partícula antes de un encuentro posee una velocidad $\mathbf{v}$ y después del encuentro adquiere una velocidad $\mathbf{v}^{\prime}$, es obvio que el cambio de velocidad debido al encuentro altera el estado dinámico de la partícula, saliendo del estado dinámico en consideración. De la misma manera consideremos una partícula que posee una velocidad $\mathbf{v}^{\prime}$ antes de un encuentro y velocidad $\mathbf{v}$ después del encuentro, por lo tanto el estado dinámico de la partícula de la misma manera cambia, entrando al estado dinámico en consideración, por lo tanto, la variación de encontrar una partícula en un estado dinámico especifico es proporcional a la razón en la cual las partículas entran y salen del sistema dinámico considerado, esto es

$$
\frac{d f}{d t} d \mathbf{q} d \mathbf{p}=-C_{\text {out }}+C_{\text {in }}
$$

Donde $C_{\text {out }}$ es la razón a cual las partículas salen del estado dinámico considerado debido a un encuentro y $C_{i n}$ es la razón a cual las partículas entran al estado dinámico considerado debido a un encuentro.

Antes de poder determinar $C_{\text {out }}$ y $C_{\text {in }}$ debemos discutir algunos aspectos acerca de los encuentros. Supongamos dos partículas que antes de un encuentro posee una velocidad $\mathbf{v}, \mathbf{v}_{1}$ y después de la colisión $\mathbf{v}^{\prime}$ y $\mathbf{v}_{1}^{\prime}$, las leyes de conservación

$$
\begin{aligned}
& \quad m \mathbf{v}+m_{1} \mathbf{v}_{1}=m \mathbf{v}^{\prime}+m_{1} \mathbf{v}_{1}^{\prime} \\
& \frac{1}{2} m \mathbf{v} \cdot \mathbf{v}+\frac{1}{2} m_{1} \mathbf{v}_{1} \cdot \mathbf{v}_{1} \\
& =\frac{1}{2} m \mathbf{v}^{\prime} \cdot \mathbf{v}^{\prime}+\frac{1}{2} m_{1} \mathbf{v}_{1}^{\prime} \cdot \mathbf{v}_{1}^{\prime}
\end{aligned}
$$

Nos muestran que para poder encontrar las velocidades $\mathbf{v}^{\prime}$ y $\mathbf{v}_{1}^{\prime}$ después del choque, debemos de encontrar dos ecuaciones mas, dado que $\mathbf{v}^{\prime}$ y $\mathbf{v}_{1}^{\prime}$ tienen tres componentes cada una y solo tenemos cuatro ecuaciones. Si suponemos que la interacción de las dos partículas solo depende de las posiciones de las mismas y es siempre radial entonces las partículas se moverán siempre en el mismo plano dado que se puede comprobar que

$$
\frac{d \mathbf{L}}{d t}=0
$$

Esta sería la quinta ecuación, la sexta ecuación la podemos deducir de la naturaleza de la interacción que están sometidas las partículas, por ejemplo, si se da el parámetro de impacto de un encuentro se puede calcular el ángulo 
de dispersión para cierto rango, con estas seis condiciones podemos determinar las velocidades después del encuentro.

En este momento podemos introducir el concepto de sección cruzada de dispersión que es muy importante en mecánica estadística, la sección cruzada de dispersión es un área hipotética probable a la cual las partículas se desvían después de un encuentro.

Ahora, supongamos un chorro de partículas con velocidad $\mathbf{v}$ y densidad numérica $n$ que se encuentran con otro chorro de partículas con velocidades $\mathbf{v}_{1}$ y densidad numérica $n_{1}$, una partícula en el primer chorro experimenta un flujo $\mathbf{I}=\left|\mathbf{v}-\mathbf{v}_{1}\right| n_{1}$ de partículas del segundo chorro, note que $\mathbf{I}$ representa la cantidad de partículas por unidad de área que cruzan una sección transversal por unidad de tiempo, esta sección transversal puede ser representada por una superficie aparente que abarca un ángulo solido $d \boldsymbol{\Omega}$, el producto de $\mathbf{I} \cdot d \boldsymbol{\Omega}$ es entonces el numero de partículas que cruzan el ángulo solido $d \boldsymbol{\Omega}$ por unidad área por unidad de tiempo. Es evidente que el número de encuentros por unidad de volumen por unidad de tiempo entre los dos grupos es proporcional a la cantidad de partículas por unidad de volumen del primer chorro y proporcional a $\mathbf{I} \cdot d \boldsymbol{\Omega}$, siendo la constante de proporcionalidad la sección cruzada de dispersión, esto es:

$$
\delta \eta=\sigma\left(\mathbf{v}, \mathbf{v}_{1}, \mathbf{v}^{\prime}, \mathbf{v}_{1}^{\prime}\right) n\left|\mathbf{v}-\mathbf{v}_{1}\right| n_{1} \cdot d \boldsymbol{\Omega}
$$

Hay que notar que $\sigma$ depende de las velocidades de las partículas antes y después del encuentro. Puede llegarse a un resultado parecido si se hubiera considerado la situación contraria y tendríamos que

$$
\delta \eta^{\prime}=\sigma\left(\mathbf{v}, \mathbf{v}_{1}, \mathbf{v}^{\prime}, \mathbf{v}_{1}^{\prime}\right) n^{\prime}\left|\mathbf{v}^{\prime}-\mathbf{v}_{1}^{\prime}\right| n_{1}^{\prime} \cdot d \boldsymbol{\Omega}
$$

Evaluemos ahora los términos $C_{\text {out }}$ y $C_{\text {in }}$. Note que según la ecuación (29)

$$
\begin{aligned}
n & =\int f(\mathbf{q}, \mathbf{v}, t) d \mathbf{v} \\
n_{1} & =\int f\left(\mathbf{q}, \mathbf{v}_{1}, t\right) d \mathbf{v}_{1} \\
n^{\prime} & =\int f\left(\mathbf{q}, \mathbf{v}^{\prime}, t\right) d \mathbf{v}^{\prime}
\end{aligned}
$$

$$
n_{1}^{\prime}=\int f\left(\mathbf{q}, \mathbf{v}_{1}^{\prime}, t\right) d \mathbf{v}_{1}^{\prime}
$$

Para cierto elemento de volumen en el espacio de las velocidades.

Si sustituimos las ecuaciones anteriores en las ecuaciones 36, 37, tenemos

$$
\begin{aligned}
\delta \eta= & \sigma\left(\mathbf{v}, \mathbf{v}_{1}, \mathbf{v}^{\prime}, \mathbf{v}_{1}^{\prime}\right) f(\mathbf{q}, \mathbf{v}, t) \\
& \cdot d \mathbf{v}\left|\mathbf{v}-\mathbf{v}_{1}\right| f\left(\mathbf{q}, \mathbf{v}_{1}, t\right) d \mathbf{v}_{1} \cdot d \boldsymbol{\Omega} \\
\delta \eta^{\prime}= & \sigma\left(\mathbf{v}, \mathbf{v}_{1}, \mathbf{v}^{\prime}, \mathbf{v}_{1}^{\prime}\right) f\left(\mathbf{q}, \mathbf{v}^{\prime}, t\right) \\
& d \mathbf{v}^{\prime}\left|\mathbf{v}^{\prime}-\mathbf{v}_{1}^{\prime}\right| f\left(\mathbf{q}, \mathbf{v}_{1}^{\prime}, t\right) d \mathbf{v}_{1}^{\prime} \cdot d \boldsymbol{\Omega}
\end{aligned}
$$

Entonces la razón a la cual las partículas salen del estado dinámico considerado es $\delta \eta$ integrada en todo el volumen $d \mathbf{v}_{1}$ y sobre todo $\Omega$, esto es

$$
\begin{aligned}
C_{\text {out }}^{\prime}= & d \mathbf{q} d \mathbf{v} \int d \mathbf{v}_{1} \int d \boldsymbol{\Omega} \cdot \sigma\left(\mathbf{v}, \mathbf{v}_{1}, \mathbf{v}^{\prime}, \mathbf{v}_{1}^{\prime}\right) \\
& \cdot\left|\mathbf{v}-\mathbf{v}_{1}\right| f(\mathbf{q}, \mathbf{v}, t) f\left(\mathbf{q}, \mathbf{v}_{1}, t\right)
\end{aligned}
$$

Siendo

$$
\begin{aligned}
& C_{\text {out }}=m C_{\text {out }}^{\prime} \\
&= m d \mathbf{q} d \mathbf{v} \int d \mathbf{v}_{1} \int d \boldsymbol{\Omega} \\
& \cdot \sigma\left(\mathbf{v}, \mathbf{v}_{1}, \mathbf{v}^{\prime}, \mathbf{v}_{1}^{\prime}\right)\left|\mathbf{v}-\mathbf{v}_{1}\right| \\
& \cdot f(\mathbf{q}, \mathbf{v}, t) f\left(\mathbf{q}, \mathbf{v}_{1}, t\right) \\
&= d \mathbf{q} d(m \mathbf{v}) \int d \mathbf{v}_{1} \int d \boldsymbol{\Omega} \\
& \cdot \sigma\left(\mathbf{v}, \mathbf{v}_{1}, \mathbf{v}^{\prime}, \mathbf{v}_{1}^{\prime}\right)\left|\mathbf{v}-\mathbf{v}_{1}\right| \\
& \cdot f(\mathbf{q}, \mathbf{v}, t) f\left(\mathbf{q}, \mathbf{v}_{1}, t\right) \\
& C_{\text {out }}= d \mathbf{q} d \mathbf{p} \int d \mathbf{v}_{1} \int d \boldsymbol{\Omega} \cdot \sigma\left(\mathbf{v}, \mathbf{v}_{1}, \mathbf{v}^{\prime}, \mathbf{v}_{1}^{\prime}\right) \\
& \cdot\left|\mathbf{v}-\mathbf{v}_{1}\right| f(\mathbf{q}, \mathbf{v}, t) f\left(\mathbf{q}, \mathbf{v}_{1}, t\right)
\end{aligned}
$$

De la misma manera podemos encontrar $C_{i n}$

$$
\begin{aligned}
C_{i n}^{\prime}= & d \mathbf{q} d \mathbf{v}^{\prime} \int d \mathbf{v}_{1}^{\prime} \int d \boldsymbol{\Omega} \cdot \sigma\left(\mathbf{v}, \mathbf{v}_{1}, \mathbf{v}^{\prime}, \mathbf{v}_{1}^{\prime}\right) \\
\cdot & \mid \mathbf{v}^{\prime}-\mathbf{v}_{1}^{\prime} f\left(\mathbf{q}, \mathbf{v}^{\prime}, t\right) f\left(\mathbf{q}, \mathbf{v}_{1}^{\prime}, t\right) \\
C_{i n}= & d \mathbf{q} d \mathbf{p}^{\prime} \int d \mathbf{v}_{1}^{\prime} \int d \boldsymbol{\Omega} \cdot \sigma\left(\mathbf{v}, \mathbf{v}_{1}, \mathbf{v}^{\prime}, \mathbf{v}_{1}^{\prime}\right) \\
& \cdot \mid \mathbf{v}^{\prime}-\mathbf{v}_{1}^{\prime} f\left(\mathbf{q}, \mathbf{v}^{\prime}, t\right) f\left(\mathbf{q}, \mathbf{v}_{1}^{\prime}, t\right)
\end{aligned}
$$


Sustituyendo la ecuación 41 y 42 en 32 , tenemos que

$$
\begin{aligned}
& \frac{d f}{d t} d \mathbf{q} d \mathbf{p}=-C_{\text {out }}+C_{\text {in }} \\
& =-d \mathbf{q} d \mathbf{p} \int d \mathbf{v}_{1} \int d \boldsymbol{\Omega} \\
& \cdot \sigma\left(\mathbf{v}, \mathbf{v}_{1}, \mathbf{v}^{\prime}, \mathbf{v}_{1}^{\prime}\right)\left|\mathbf{v}-\mathbf{v}_{1}\right| f(\mathbf{q}, \mathbf{v}, t) f\left(\mathbf{q}, \mathbf{v}_{1}, t\right) \\
& +d \mathbf{q} d \mathbf{p}^{\prime} \int d \mathbf{v}_{1}^{\prime} \int d \boldsymbol{\Omega} \cdot \sigma\left(\mathbf{v}, \mathbf{v}_{1}, \mathbf{v}^{\prime}, \mathbf{v}_{1}^{\prime}\right) \\
& \left|\mathbf{v}^{\prime}-\mathbf{v}_{1}^{\prime}\right| f\left(\mathbf{q}, \mathbf{v}^{\prime}, t\right) f\left(\mathbf{q}, \mathbf{v}_{1}^{\prime}, t\right)
\end{aligned}
$$

Y dado que por el teorema de Liouville y la ecuación de conservación 33 .

$$
\begin{aligned}
d \mathbf{v} d \mathbf{v}_{1} & =d \mathbf{v}^{\prime} d \mathbf{v}_{1}^{\prime} \\
\left|\mathbf{v}-\mathbf{v}_{1}\right| & =\left|\mathbf{v}^{\prime}-\mathbf{v}_{1}^{\prime}\right|
\end{aligned}
$$

Entonces la ecuación de Boltzman considerando las colisiones queda de la siguiente manera:

$$
\begin{gathered}
\frac{d f}{d t}=\int d \mathbf{v}_{1} \int d \boldsymbol{\Omega} \cdot \sigma\left(\mathbf{v}, \mathbf{v}_{1}, \mathbf{v}^{\prime}, \mathbf{v}_{1}^{\prime}\right) \\
\left|\cdot \mathbf{v}-\mathbf{v}_{1}\right|\left(f^{\prime} f_{1}^{\prime}-f f_{1}\right)
\end{gathered}
$$

Donde

$$
\begin{aligned}
& f^{\prime}=f\left(\mathbf{q}, \mathbf{v}^{\prime}, t\right) \\
& f_{1}^{\prime}=f\left(\mathbf{q}, \mathbf{v}_{1}^{\prime}, t\right) \\
& f=f(\mathbf{q}, \mathbf{v}, t) \\
& f_{1}=f\left(\mathbf{q}, \mathbf{v}_{1}, t\right)
\end{aligned}
$$

Si colocamos la ecuación 21 en el espacio $x y z$ la ecuación 43 queda de la siguiente forma:

$$
\begin{aligned}
& \frac{\partial f}{\partial t}+\frac{\partial f}{\partial \mathbf{r}} \cdot \mathbf{v}+\frac{\partial f}{\partial \mathbf{v}} \cdot \frac{\mathbf{F}}{m} \\
& =\int d \mathbf{v}_{1} \int d \boldsymbol{\Omega} \cdot \sigma\left(\mathbf{v}, \mathbf{v}_{1}, \mathbf{v}^{\prime}, \mathbf{v}_{1}^{\prime}\right) \\
& \cdot\left|\mathbf{v}-\mathbf{v}_{1}\right|\left(f^{\prime} f_{1}^{\prime}-f f_{1}\right)
\end{aligned}
$$

La integral a la derecha de la ecuación anterior se conoce como la integral de colisiones.

\section{Postulado de GibBs y ECUACIONES DE TRANSPORTE}

Ya hemos dicho que tratar de seguir el movimiento de cada una de las partículas, es algo que no se puede hacer, pero ya hemos definido el concepto de ensamble, el cual nos ayuda a solucionar dicho problema.

Supongamos que tenemos un gas diluido constituido por $l$ partículas, y que existen $j$ partículas de una especie, cada una de ellas tiene una velocidad $\mathbf{v}_{j}$ y una posición $\mathbf{r}_{j}$ en un tiempo especifico $t$, entonces si asociamos una cantidad $\phi\left(\mathbf{r}_{j}, \mathbf{v}_{j}, t\right)$ a una partícula que se encuentra en una posición $\mathbf{r}_{j}$ y tiene una velocidad $\mathbf{v}_{j}$ en un tiempo $t$, entonces el valor promedio de $\phi$ para todas la partículas en la cercanía de $\mathbf{r}_{j}$ en el mismo instante $t$, esta dado por (Ver $[8][3]$, 7 )

$$
\begin{aligned}
& <\phi\left(\mathbf{r}_{j}, \mathbf{v}_{j}, t\right)> \\
& =\frac{1}{n} \int \phi\left(\mathbf{r}_{j}, \mathbf{v}_{j}, t\right) f\left(\mathbf{r}_{j}, \mathbf{v}_{j}, t\right) d \mathbf{v}_{j}
\end{aligned}
$$

donde $n$ es la densidad numérica presentada en el capítulo 1. El enunciado anterior es precisamente el postulado de Gibbs, el cual nos permitirá conocer algunas propiedades de un gas diluido. Hay que notar que solo estas cantidades promedios son susceptibles de medición.

Sea $\mathbf{v}_{j}$ la velocidad de una partícula o molécula de una especie $j$ según un sistema de referencia, la velocidad promedio de la partícula entonces esta dada por

$$
\mathbf{c}_{j}=\frac{1}{n} \int \mathbf{v}_{j} f\left(\mathbf{r}_{j}, \mathbf{v}_{j}, t\right) d \mathbf{v}_{j}
$$

y la cual representa la velocidad de flujo macroscópico de las moléculas de especie $j$, si tomamos en consideración todas las moléculas, de todas las especies del gas, podemos definir la velocidad de masa promedio como

$$
\mathbf{c}_{0}=\frac{\sum_{j} \rho_{j} \mathbf{c}_{j}}{\sum_{j} \rho_{j}}
$$

esta velocidad es también llamada velocidad de flujo de gas.

También podemos definir la velocidad peculiar de la forma

$$
\mathbf{C}_{j}=\mathbf{c}_{j}-\mathbf{c}_{0}
$$


encontrando el promedio de la velocidad peculiar

$$
<\mathbf{C}>=\frac{1}{n} \int \mathbf{C}_{j} f\left(\mathbf{r}_{j}, \mathbf{v}_{j}, t\right) d \mathbf{v}_{j}
$$

que se conoce como velocidad de difusión, que representa la velocidad promedio relativa de las partículas dentro del gas según la velocidad de flujo del mismo.

La energía cinética de una partícula con velocidad $\mathbf{c}_{j}$ es

$$
\frac{1}{2} m_{j} c_{j}^{2}=\frac{1}{2} m_{j} C_{j}^{2}+\frac{1}{2} m_{j} c_{0}^{2}
$$

en donde el segundo término de la derecha es la energía cinética del flujo y el primer termino de la derecha se le da el nombre de energía interna, por lo tanto la energía interna es la energía cinética que resulta de la desviación de las velocidades moleculares según la velocidad de flujo, así, la energía interna promedio sera

$$
<U_{j}>=\frac{1}{n} \int \frac{1}{2} m_{j} C^{2} f\left(\mathbf{r}_{j}, \mathbf{v}_{j}, t\right) d \mathbf{v}_{j}
$$

También podemos asociar a una partícula una cantidad $\phi(\mathbf{r}, \mathbf{v}, t)$ y una dirección $\hat{e}$, un flujo de $\phi$ en la dirección de $\hat{e}$.

supongamos que una partícula de especie $j$ tiene una velocidad $\mathbf{c}_{j}$ cruza una superficie del flujo de gas, la cual se mueve a una velocidad $\mathbf{c}_{0}$, en cierto tiempo $d t$ la partícula a barrido un volumen en la dirección de ê igual a

$$
\left(\mathbf{c}_{j}-\mathbf{c}_{0}\right) \cdot d t d \mathbf{a}
$$

donde $d a$ es una superficie del flujo de gas que tiene una dirección $\hat{e}$, el numero de partículas dentro del volumen es simplemente

$$
\mathbf{C}_{j} d t d a \cdot \hat{e} f\left(\mathbf{r}_{j}, \mathbf{v}_{j}, t\right) d \mathbf{v}_{j}
$$

por lo tanto el flujo de $\phi$ asociado a la partícula o molécula dentro del volumen es

$$
\mathbf{C}_{j} \phi\left(\mathbf{r}_{j}, \mathbf{v}_{j}, t\right) f\left(\mathbf{r}_{j}, \mathbf{v}_{j}, t\right) d \mathbf{v}_{j} \cdot \hat{e} d t d a
$$

si dividimos entre $d t d a$ e integramos en todo el espectro de velocidades encontramos el flujo de $\phi$ en la dirección de $\hat{e}$

$$
\begin{aligned}
& n<\phi\left(\mathbf{r}_{j}, \mathbf{v}_{j}, t\right)>\cdot \hat{e} \\
& =\int \mathbf{C}_{j} \phi\left(\mathbf{r}_{j}, \mathbf{v}_{j}, t\right) f\left(\mathbf{r}_{j}, \mathbf{v}_{j}, t\right) d \mathbf{v}_{j} \cdot \hat{e}
\end{aligned}
$$

y por lo tanto el flujo de $\phi$ es

$$
\mathbf{E}_{j}=\int \mathbf{C}_{j} \phi\left(\mathbf{r}_{j}, \mathbf{v}_{j}, t\right) f\left(\mathbf{r}_{j}, \mathbf{v}_{j}, t\right) d \mathbf{v}_{j}
$$

\section{Transporte de masa}

Aquí $\phi=m_{j}$ (Ver [8]), sustituyendo en la ecuación 2.12, tenemos

$$
\begin{aligned}
\mathbf{E} & =\int \mathbf{C}_{j} m_{j} f\left(\mathbf{r}_{j}, \mathbf{v}_{j}, t\right) d \mathbf{v}_{j} \\
& =n_{j} m_{j}\left(\frac{1}{n_{j}} \int \mathbf{C}_{j} f\left(\mathbf{r}_{j}, \mathbf{v}_{j}, t\right) d \mathbf{v}_{j}\right) \\
& =\rho_{j}<\mathbf{C}_{j}>
\end{aligned}
$$

podemos notar que la ecuación 2.15 concuerda con un flujo por unidad de área de un fluido ideal.

\section{Transporte de momento}

Aquí $\phi=m_{j} \mathbf{C}_{j}($ Ver [8] $)$

$$
\begin{aligned}
\mathrm{P} & =\int \mathbf{C}_{j}\left(m_{j} \mathbf{C}_{j}\right) f\left(\mathbf{r}_{j}, \mathbf{v}_{j}, t\right) d \mathbf{v}_{j} \\
& =n_{j} m_{j}\left(\frac{1}{n_{j}} \int \mathbf{C}_{j} \mathbf{C}_{j} f\left(\mathbf{r}_{j}, \mathbf{v}_{j}, t\right) d \mathbf{v}_{j}\right) \\
& =\rho_{j}\left\langle\mathbf{C}_{j} \mathbf{C}_{j}\right\rangle
\end{aligned}
$$

a esto se le da el nombre de tensor de presiones, podemos notar que la presión en una superficie dada, orientada con $\hat{e}$ es

$$
\mathrm{P} \cdot \hat{e}=p=\rho_{j}<\mathbf{C}_{j} \mathbf{C}_{j}>\cdot \hat{e}
$$

con

$$
<\mathbf{C}_{j} \mathbf{C}_{j}>=\left(\begin{array}{ccc}
C_{x j}^{2} & C_{x j} C_{y j} & C_{x j} C_{z j} \\
C_{y j} C_{x j} & C_{y j}^{2} & C_{y j} C_{z j} \\
C_{z j} C_{x j} & C_{z j} C_{y j} & C_{z j}^{2}
\end{array}\right)
$$


III. Transporte de energía

Aquí $\phi=\frac{1}{2} m_{j} \mathbf{c}_{j}^{2}$ (Ver [] $)$

$$
\begin{aligned}
\mathbf{q} & =\int \mathbf{C}_{j}\left(\frac{1}{2} m_{j} \mathbf{C}_{j}^{2}\right) f\left(\mathbf{r}_{j}, \mathbf{v}_{j}, t\right) d \mathbf{v}_{j} \\
& =\frac{1}{2} n_{j} m_{j}\left(\frac{1}{n_{j}} \int C_{j}^{2} \mathbf{C}_{j} f\left(\mathbf{r}_{j}, \mathbf{v}_{j}, t\right) d \mathbf{v}_{j}\right) \\
& =\frac{1}{2} \rho_{j}<C_{j}^{2} \mathbf{C}_{j}>
\end{aligned}
$$

que se le da el nombre de vector de flujo de calor.

\section{REFERENCIAS}

[1] Joseph O. Hirschfelder \& Charles F. Curtiss \& R. Byron Bird. Molecular theory of gases and liquids. Wiley, 1964.
[2] M. G. Calkin. Lagrangian and Hamiltonian Mechanics. World Scientific, 1996.

[3] S. Chapman \& T.G. Cowling. The mathematical theory of non-uniform gases. Cambridge Mathematical Library, 1995.

[4] Herbert Goldstein. Macánica Clásica. Tolle, Lege, 1972.

[5] Joseph Norwood Jr. Mecánica Clásica a nivel intermedio. Prentice Hall, 1981.

[6] L. D. Landau and E. M. Lifshitz. Statistical Physics Part 1. Elsevier, 1980.

[7] L. D. Landau and E. M. Lifshitz. Physical Kinetics. Elsevier, 1981.

[8] Donald A. McQuarrie. Statistical Mechanics. University science book, 2000. 